\title{
Improvement of the LNE's metrological Atomic Force Microscope (mAFM) performance: Design of new mAFM head dedicated for nanometrology applications
}

\author{
Younes Boukellal $^{1, \text { a }}$, Sébastien Ducourtieux ${ }^{1}$ and Benoit Poyet ${ }^{1}$ \\ ${ }^{1}$ Laboratoire National de métrologie et d'Essais, 29, Avenue Roger Hennequin 78190 Trappes, France
}

\begin{abstract}
A metrological Atomic Force Microscope (mAFM) has been developed at LNE [1, 2]. It is mainly used for performing traceable measurement and calibration of transfer standards dedicated to scanning probe and scanning electron microscopes. In order to improve the mAFM performance and reduce the measurement uncertainty, a new mAFM head is being developed and will be integrated on the instrument. It consists of an immobile AFM head working in a zero detection mode. The head is kinematically mounted on the stationary part of a home-made piezo-actuated flexure stage that produces three translations with a displacement range of $60 \mu \mathrm{m}$ along $X$ and $Y$ axes and $15 \mu \mathrm{m}$ along $Z$ axis. The tip-sample relative position is measured with four dual pass differential interferometers with an expected uncertainty of about $1 \mathrm{~nm}$. This paper presents the design of new $\mathrm{mAFM}$ and the evaluation of the first uncertainty components of the instrument.
\end{abstract}

\section{The AFM head}

Up to now, a commercial AFM head (Park Instruments, XE-100 AFM head [3]) with a non-optimized metrology frame is used. This frame is about $10 \mathrm{~cm}$ long mainly composed of aluminium and of course sensitive to thermal drift. The tip is directly linked to this frame (figure 1-a). First characterization of the instrument equipped with this AFM head revealed an unwanted drift close to $10 \mathrm{~nm}$ during a scan of 11 hours on a reference
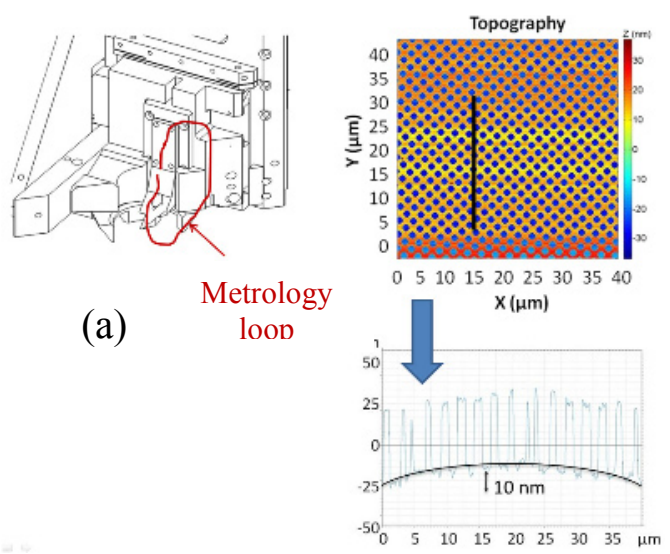

Figure 1. Topography drift (z direction) measured during the scan of a reference sample. (a) Identification of the metrology loop of the commercial AFM head (b) Sample image produced by recording XYZ positions measured with interferometers. (c) Topography drift along $Z$ axis.
The drift is mainly due to thermal dilatation of the $\mathrm{Z}$ metrology frame inside the AFM head. The head design also presents other drawbacks as for example the two thermal sources contained inside, the one associated to the laser diode used for the detection of cantilever deflections and the one produced by the electronic circuit used for the conditioning of the signal generated by the quad cell photodiode. Mainly for these reasons, we have started the development of a new metrological AFM head with no heat source. The most important requirements of the design are:

- An immobile AFM head working in a zero detection mode.

- A resolution of $0.1 \mathrm{~nm}$ for the cantilever deflection measurement with a low noise level below $1 \mathrm{~nm}$.

- An optical access to observe and position the tip with respect to the sample using an optical microscope.

- A tip positioned at the thermal center of the instrument.

- A tip/sample approach system with no heat generation.

\footnotetext{
${ }^{\mathrm{a}}$ Corresponding author: Younes.boukellal@lne.fr
} 


\subsection{The cantilever detection system}

The accuracy and the stability for measuring the cantilever displacement are very important for reaching a good resolution of mAFM deflexion detection. Many techniques have been developed for this measurement based on different physical principles (optical beam, optical interferometer, tuning fork, capacitive cantilever, piezo-resistive cantilever...). Our cantilever detection system is based on the optical beam deflexion method (OBD) [4], with some specific modifications in order to eliminate the major heat sources around the tip. The system and the associated characterization results will be discussed in poster

Figure 1. The mAFM cantilever deflexion detection system (Sectional view) with (1) a tip/tilt stage, (2) the super luminescent diode, (3) a reflecting prism, (4) a reflecting mirror, (5) the home-made deflexion sensitive detector, (6) the microscope objective field of view, (7) the cantilever/tip holder.

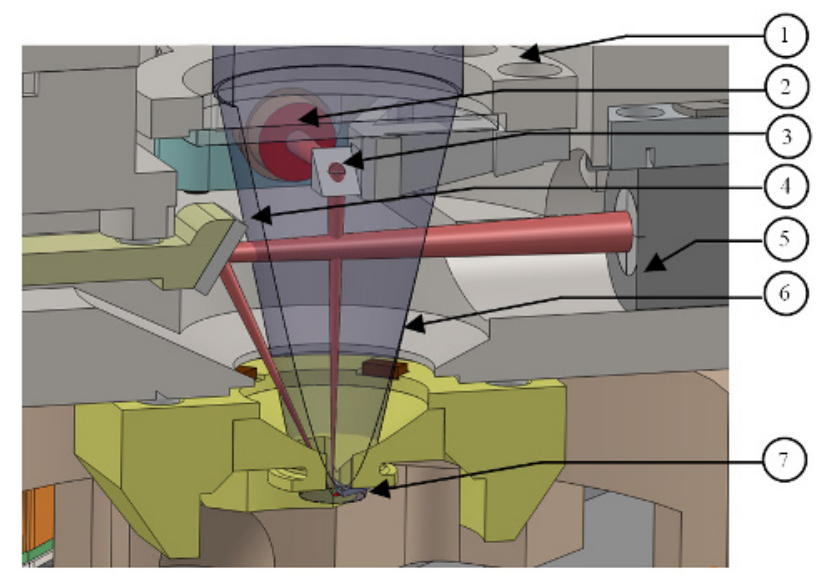

We decided to use a fibered super luminescent diode (SLD) (figure 1-(2)) to reduce the coherence length of the light, thus reducing the optical parasitic signal due to optical interference. The SLD has a center wavelength of $681.2 \mathrm{~nm}$, a spectral width of $9.6 \mathrm{~nm}$ and very small coherence length $(28 \mu \mathrm{m})$ which is an order of magnitude smaller than the coherence length of typical laser diode. The SLD is equipped with a Faraday isolator to attenuate the back reflected light to the SLD. Temperature is stabilized by peltier element which is integrated in the SLD cage. The SLD cage is deported outside the mAFM room. The light beam from the SLD fiber is reflected by a prism (figure 1-(3)) and deflected onto the cantilever (figure 1-(7)). The whole (prism + SLD) are linked to a tip/tilt stage (figure 1-(1)) to control the position of the beam on the cantilever and to always maintain the spot centered on the prism. The spot size on the backside of the cantilever is equal to $23 \mu \mathrm{m}$ and the working distance is $58 \mathrm{~mm}$ which frees more space under the SLD to integrate the OBD components. From the cantilever, the reflected beam is directed by a mirror (figure 1-(4)) to a home-made deflexion sensitive detector (figure 1-(5)). The detector axes are aligned with the cantilever axes. In order to observe the spot on the cantilever we use a long working distance microscope objective (figure 1-(6)) $(\mathrm{WD}=51 \mathrm{~mm}, \mathrm{x} 10, \mathrm{ON} 0.21)$.

\subsection{The mirror blocks}

Figure 2. The mAFM mirror blocks configuration with (1) the mAFM head, (2) the reference prism, (3) the cantilever + tip, (4) a mirror faces, (5) the interferometer beams, (6) the measurement prism, (7) the $\mathrm{Z}$ stage

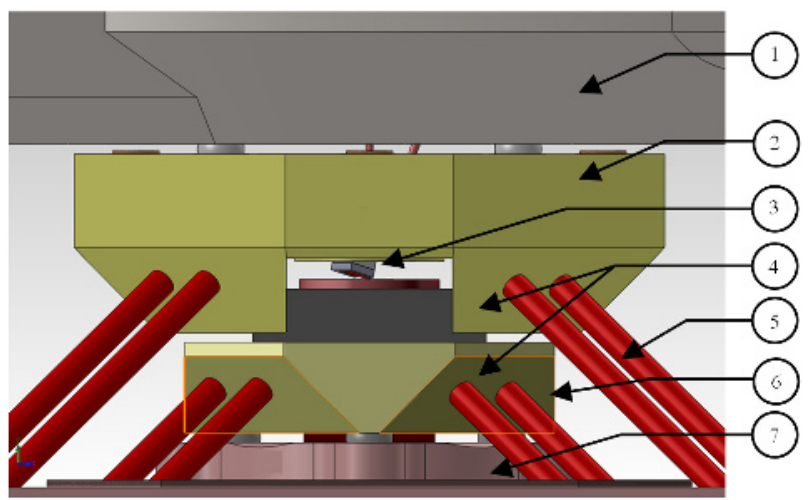

To measure the relative displacement of the sample with regards of the tip, the differential interferometers aim at mirrors (figure 2-(5)) directly machined on two prisms made of Zerodur ${ }^{\circledR}$ to minimize errors introduced by thermal expansion. Due to the system geometry, the two blocks have the shape of a truncated inverted four-face pyramid with a half top angle of 55०. The mirrors are machined and polished to obtain flatness better than $\lambda / 10$ and an orthogonality deviation of less than $300 \mu \mathrm{rad}$ (figure 2-(4)). The first prism (figure2-(2)) is linked to the AFM head using v-groove kinematic coupling. It constitutes the reference mirror block for the interferometers. The tip (figure 2-(3)) is directly linked to the reference prism which shortens the metrology loop length. The second one, the measurement mirror block (figure 2-(6)), is associated with the sample displacement. It is linked to the $Z$ stage (figure 2-(7)) using a v-groove kinematic coupling. In this configuration, the whole of the system is Abbe compliant.

\section{3 mAFM head support frame and the tip/sample approach frame}

Figure 3. The mAFM head and its support frame with (1) a vgroove support, (2) the mAFM head support frame, (3) the piezo-legs motors, (4) The tip sample approach frame.

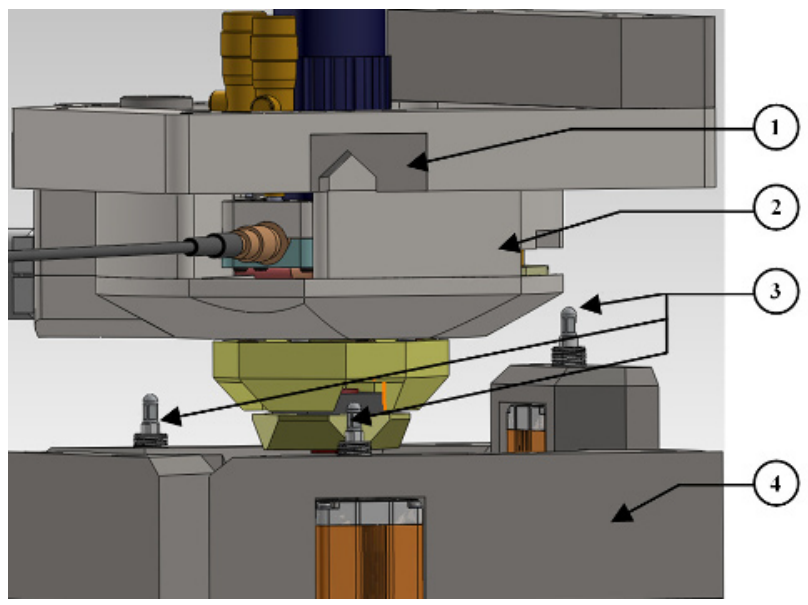


Both of the support frame (figure 3-(2)) and the tip/sample approach frame (figure 3-(4)) are made from invar. The two structures play an important role in the thermal design strategy. Indeed the large amount of invar allows us to improve the thermal inertia and thermal stability of the setup. Furthermore, the tip/sample approach frame supports three piezo-legs motors (no heat generation) (figure 3-(3)) with an integrated linear encoder. The translations of these motors are first combined in order to generate the necessary rotations for the alignment of the reference mirror blocks in regard with the measurement mirror block. Once the alignment of the mirrors performed, the motors are synchronized to generate a vertical translation and thus performed the tip/sample approach with high resolution steps. The mAFM head is linked to the motors using three v-groove kinematic coupling (figure 3-(1)).

A more detailed description of the mAFM head will be presented on the poster

\section{Evaluation of the first uncertainty components}

\subsection{Evaluation of the translation stage parasitic rotations}

The three axis translation stages used on the LNE's mAFM has been characterized to determine the impact of Abbe error in the uncertainty budget. To evaluate in situ the three parasitic rotations of the translation stage, we developed a dedicated measurement bench.

Figure 4. measurement bench for parasitic rotation evaluation

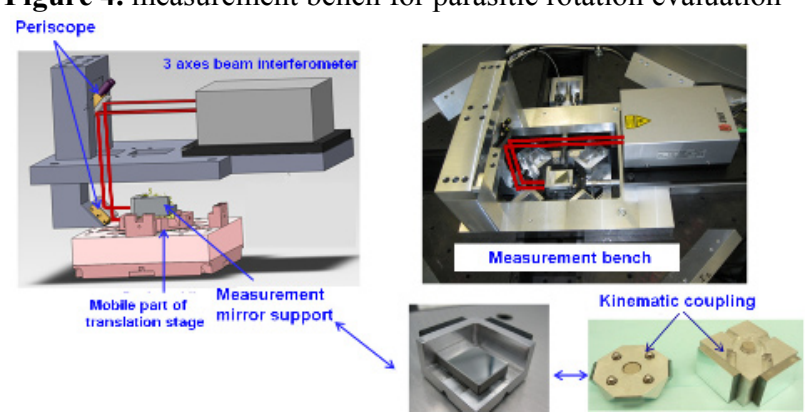

It consists of a triple beam plane mirror interferometer (SIOS, SP-TR Series, with $0.1 \mathrm{~nm}$ resolution for length measurement and $0.01 \mu \mathrm{rad}$ for angular measurement) and a mirror holder kinematically mounted on the sample holder. Because the interferometer is capable of performing two rotation measurements, the pitch and yaw rotations have been measured simultaneously in a first configuration and the roll rotation was measured in a second one. The parasitic rotations were evaluated while the stage was controlled in close loop using the mAFM interferometers. Results are summarized in table 1.
Table 1. Parasitic rotations of the XYZ translation stage

\begin{tabular}{|c|c|c|c|c|c|c|c|c|c|}
\hline Absolute & \multicolumn{3}{|c|}{$T_{x}$} & \multicolumn{3}{|c|}{$T_{y}$} & \multicolumn{3}{|c|}{$T_{z}$} \\
\hline $\begin{array}{l}(\mu \mathrm{rad}) \\
60 \mu \mathrm{m} \mathrm{x}\end{array}$ & $R_{x}$ & $R_{Y}$ & $R_{Z}$ & $R_{X}$ & $R_{Y}$ & $R_{Z}$ & $R_{X}$ & $R_{Y}$ & $R_{Z}$ \\
\hline $\begin{array}{c}60 \mu \mathrm{m} x \\
15 \mu \mathrm{m}\end{array}$ & 0.6 & 6 & 0.7 & 5.5 & 0.5 & 0.7 & 4.2 & 0.7 & 2.2 \\
\hline
\end{tabular}

Even though the measured rotations are linear and repeatable (corrections will be possible), they are higher than the expected $1 \mu \mathrm{rad}$ and higher than the one previously measured on a stage prototype [2]. Many effects has been identified to explain some of the highest parasitic rotational motions measured for Tx Ry and Ty $\mathrm{Rx}$ - see Table 1: (i) the location of the piezo actuators with respect to the guidance mechanism, (ii) a lack of decoupling between the $\mathrm{X}, \mathrm{Y}$ and $\mathrm{Z}$ axis and (iii) a defect on the flexure stage assembly. It will be presented as well as the solutions under investigation.

\subsection{Evaluation of interferometer measurement stability}

We've experimented that the differential interferometer measurements in ambient air are sensitive to differential variations of air index between the two arms. Fig. 1 shows that the noise level associated to air index differential fluctuation is about $20 \mathrm{~nm}$ peak-to-peak without any specific protections. On the other hand, thanks to an aluminium enclosure along the beam path, the air index is more homogeneous between the two arms and more stable over long time measurements. Thus, the equivalent noise level is reduced with a ratio of 40 to 0.5 nm peak-to-peak (see Figure 4).

Figure 5. (up) interferometer stability measurements, (down) Allan deviation with the protection.
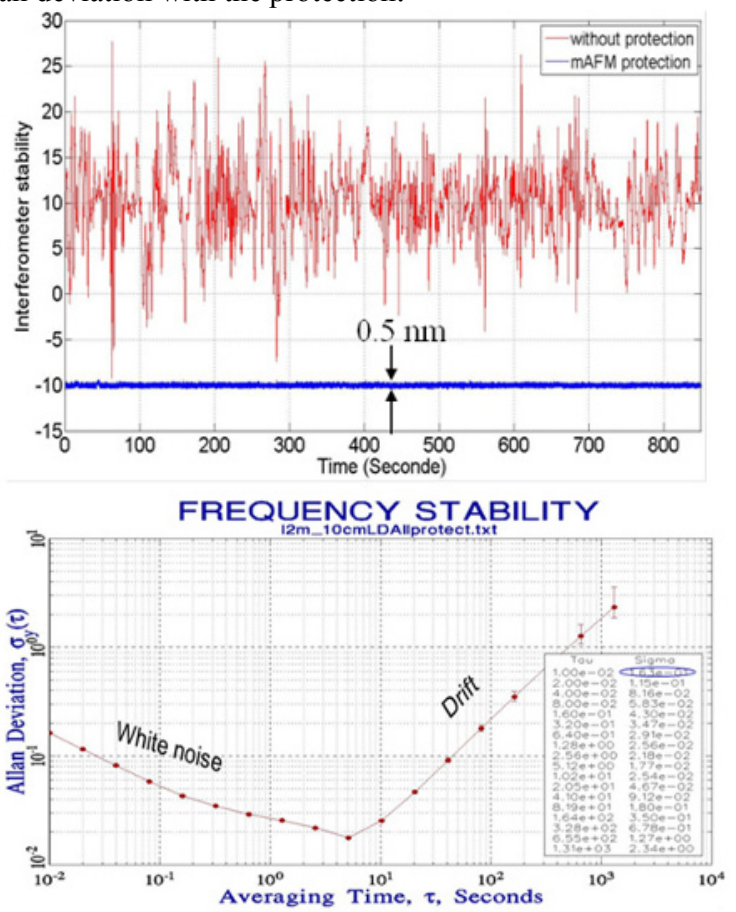
We evaluated the noise level the standard deviation when the beams were protected by using two reference methods: Allan variance and power spectral density. The two methods gave the same experimental standard deviation value, equal to $0.163 \mathrm{~nm}$, which validates the measurement principle [5]. We also evaluated the long term position stability over 50 hours with the protection. The Allan deviation vs. averaging time shows that below 5 seconds, the noise nature is white. Over 5 seconds, the drift becomes predominant. This experiment has shown that the beam path enclosure improves the interferometer measurement stability

\subsection{Evaluation of the interferometer non-linearity}

The four differential interferometers have nonlinearity behaviour due to polarisation mixing mainly caused by misalignment and imperfection of optics used in the interferometer head. To evaluate its nonlinearities, we implemented an experimental setup which comprises an interferometer module from Renishaw (RLD-X3-DI) with two mirrors. The first one is attached to the $\mathrm{XYZ}$ translation stage (Physik Instrumente P-517C), the second one is linked to the mAFM tip. A $20 \mathrm{~mm}$ thick aluminium enclosure reduces the effect of air index fluctuations and the interferometer drift during the experiment. To minimize the error induced by air turbulence, the path length of the interferometer is as short as possible $(10 \mathrm{~cm})$. A triangle wave is applied to the stage controller to provide fine displacement in the range of several micrometers along the interferometer axis. The measurements of the stage capacitive sensor are also used to be compared with interferometer measurements. As shown on Figure 3, the nonlinearity is determined by measuring the residual displacement when the first order of the interferometer versus capacitive sensor curve is removed.

Figure 6. Interferometer nonlinearity (left) and its associated distribution (right)

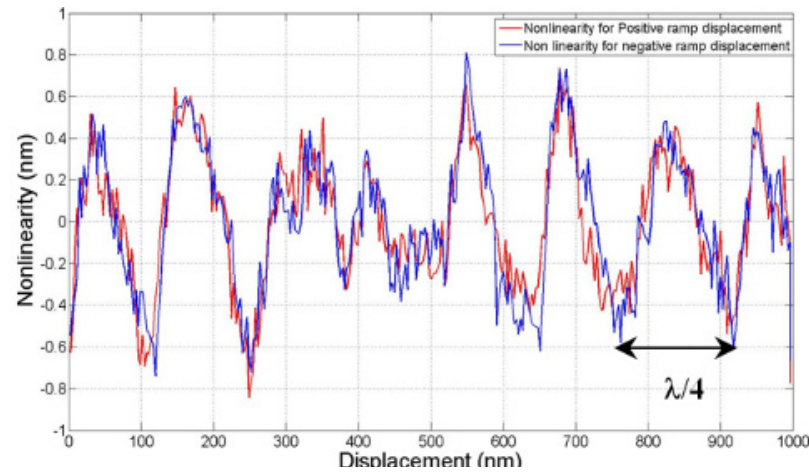

The Lissajous representation (sine vs. cosine signal) gives information about the quadrature signals quality of interferometers. For a $100 \%$ Lissajous strength, the nonlinearity is $1.41 \mathrm{~nm}$ peak-to-peak. Its periodicity is consistent with the one expected for a double pass interferometer $(\lambda / 4 \approx 158 \mathrm{~nm})$. The distribution associated to the nonlinearity is normal with a standard deviation of $0.47 \mathrm{~nm}$. In order to evaluate the impact of the interferometers misalignment, we evaluated the nonlinearity error variation with respect to the Lissajous signal strength. The Lissajous signal strength must be over $50 \%$ to perform interferometric measurements with low nonlinearity error (i.e. $1.41 \mathrm{~nm}$ peak to peak). Below $50 \%$, the nonlinearity error becomes significant (i.e. more than $4 \mathrm{~nm}$ peak to peak) and does not meet the expected uncertainty requirements for the tip-sample relative position measurement. Thus, it would be useful to find an optimal alignment before starting measurements on the mAFM.

Figure 7. Non-linearity variation with respect to the optical phase shift inside the interferometer head

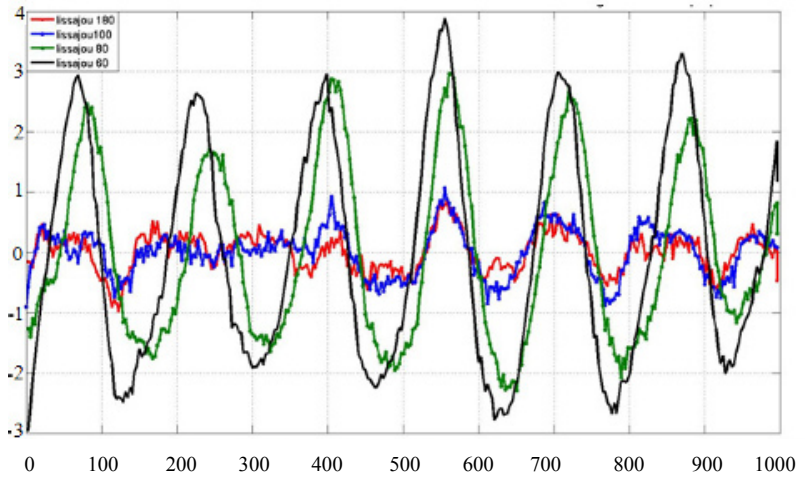

\section{Conclusion}

A new metrological AFM head with no heat sources around the tip is currently under development in order to avoid thermal drift of the instruments during scanning. The 3D design is finished and the next step will be devoted to the prototype realization.

Experimental investigations gave the first uncertainty components of the LNE's mAFM. The nonlinearity error is not critical in the uncertainty budget. The interferometer stability evaluation shows the importance of protecting them to reduce the refractive index variations between the two arms of each interferometer. To lower Abbe error to $1 \mathrm{~nm}$, new configurations for the actuation of flexure stage will be investigated and. All those experimentally determined uncertainty components will be used to modelize the mAFM measurement process and to investigate its measurement uncertainty.

\section{Bibliography}

[1] S. Ducourtieux and B. Poyet, Meas. Sci. Technol. 22 (2011) 094010

[2] B. Poyet, 2010, Conception d'un microscope à force atomique métrologique $P h D$ Thesis Université de Versailles Saint-Quentin en Yvelines

[3] Kwon J, Hong J, Kim Y S, Lee D Y, Lee K, Lee S M and Park S 2003 Atomic force microscope with improved scan accuracy, scan speed, and optical vision Rev. Sci. Instrum. 74 4378-83

[4] G. Binnig, C.F. Quate and Ch. Gerber, Phys. Rev.Lett 56(1986)930

[5] T.J. Witt, Instrumentation and Measurement, IEEE Transactions (April 2001), 50 (2), pg. 445-448 